\title{
Betanodavirus: dissection of the viral life cycle
}

\begin{abstract}
Progressive research has been recently made in dissecting the molecular biology of Betanodavirus life cycle, the causative pathogen of viral encephalopathy and retinopathy in economic important marine fish species. Establishment of betanodavirus infectious clone allows the manipulation of virus genome for functional genomic study, which elucidates the biological event of the viral life cycle at molecular level. The betanodavirus strategizes its replication by expressing anti-apoptosis/antinecrotic proteins to maintain the cell viability during early infection. Subsequently utilizes and controls the biological machinery of the infected cells for viral genome replication. Towards the late phase of infection, mass production of capsid protein for virion assembly induces the activation of host apoptosis pathway. It eventually leads to the cell lysis and death, which the lysis of cell contributes to the accomplishment of viral shedding that completes a viral life cycle. The recent efforts to dissect the entire betanodavirus life cycle are currently reviewed.
\end{abstract}

Keyword: Betanodavirus; Virus attachment and entry; Virus encapsidation and shedding; Virus life cycle; Virus replication 